\title{
FRAÇÕES DE FÓSFORO E ATIVIDADE DA FOSFATASE ÁCIDA EM PLANTAS DE FEIJ OEIRO CULTIVADAS EM SOLOS DE VÁRZEA ${ }^{(1)}$
}

\author{
L. A. FER NANDE $S^{(2)}$, V. FAQUIN ${ }^{(3)}$, \\ A. E. FURTINI NETO(3) \& N. CURI (3)
}

\begin{abstract}
RESUMO
Foi realizado um experimento em casa de vegetação do Departamento de Ciência do Solo da Universidade Federal de Lavras, com o objetivo de verificar a influência dos atributos de quatro solos de várzea sobre a distribuição de algumas frações de fósforo nas folhas de feijoeiro, determinar suas respectivas concentrações críticas e a relação dessas frações com a atividade da fosfatase ácida foliar e a relação entre a atividade da fosfatase ácida determinada "in vitro" e "in vi vo". 0 delineamento experimental foi o inteiramente casualizado, em esquema fatorial $4 \times 5 \times 2$, com quatro repetições, sendo quatro solos [Glei Húmico (GH), Orgânico (O), Aluvial (A) e Glei Pouco Húmico (GP)], cinco doses de $P\left(75,150,300,500\right.$ e $800 \mathrm{mg} \mathrm{dm}^{-3}$ de $P$ ) e dois níveis de calagem (sem e com). Cada parcela foi constituída por um vaso com três $\mathrm{dm}^{3}$, onde foram cultivadas três plantas de feijoeiro. Uma das plantas foi colhida no florescimento, determinando-se, nas folhas, as concentrações de $P$ total $(P t), P$ total solúvel em ácido (Pts), $\mathrm{P}$ orgânico (Po) e $\mathrm{P}$ inorgânico solúvel em ácido (Pi). Nas demais plantas, colhidas no final do ciclo, foi avaliada a produção de grãos. Os níveis críticos das frações de $P$ no feijoeiro cultivado nos solos GH, O, A e GP, sem calagem, foram, respectivamente: $P t=2,3,2,2,2,0$, e 1,3 $\mathrm{mg} \mathrm{g}^{-1}$; $\mathrm{Pts}=110,67,78 \mathrm{e}$ 63; Po $=32$, 19, 20 e 21 e Pi $=77,47,58$ e $43 \mu \mathrm{g} \mathrm{g}^{-1}$. Com calagem, os níveis críticos respectivos foram: $\mathrm{Pt}=2,8,3,0,3,5$ e 4,2 $\mathrm{mg} \mathrm{g}^{1}$; Pts $=105,96,130$ e 154; $\mathrm{Po}=47$, 38, 42 e $52 \mathrm{e} \mathrm{Pi}=59,58,88 \mathrm{e} 103 \mathrm{\mu g} \mathrm{g}^{-1}$. A atividade da fosfatase ácida não foi uma boa característica de diagnose nutricional de fósforo em condições de severa deficiência desse elemento.
\end{abstract}

Termos de indexação: nível crítico, nutrição de plantas, atividade enzi mática.

(1) Parte da Tese de Doutorado do primeiro autor. Recebido para publicação em fevereiro de 2000 e aprovado em junho de 2000. Trabalho Financiado pelo CNPq e FAPEMIG.

(2) Professor do Núcleo de Ciências Agrárias, Universidade Federal de Minas Gerias - UFMG. Caixa Postal 135, CEP 39404-006 Montes Claros (MG). E-mail: Iafernand@ig.com.br

(3) Professor do Departamento de Ciência do Solo, Universidade Federal de Lavras - UFLA. Caixa Postal 37, CEP 37200-000 Lavras (MG). Bolsista do CNPq. E-mails: vafaquin@ufla.br; afurtini@ufla.br; niltcuri@ufla.br 


\title{
SUMMARY: PHOSPHORUS FRACTIONS AND ACID PHOSPHATASE ACTIVITY IN BEAN PLANTS CULTIVATED IN LOWLAND SOILS
}

\begin{abstract}
An experiment was carried out under greenhouse conditions at the Soil Science Department of theUniversidadeF ederal deLavras, Minas Gerais, Brazil, to determinecritical levels of different phophorus fractions and acid phosphatase activity in bean plants (Phaseolus vulgaris L. cv. Carioca-MG); the relationship between these fractions and the acid phosphatase activity and grain production, and the relationship between acid phosphataseactivity determined "in vivo" and "in vitro". Theplants werecultivated on four Iowl and soils of southern M inas Gerais, Brazil and subjected to five phosphorus doses, with and without lime application. The experimental design was completely randomized, in a factorial scheme $4 \times 5 \times 2$ with four replications, as follows: four soils [Humic Gley $(G H)$, Bog Soil (O), Alluvial (A) and Low Humic Gley (GP)]; five P doses (75, 150, 300, 500 and $800 \mathrm{mg} \mathrm{dm}^{-3}$ ); and two levels of liming application (with and without lime). Each plot consisted of a thre $\mathrm{dm}^{-3}$ pot where thre bean plants were grown. One of the plants was harvested at fl owering with its l eaf concentrations of total P (Pt), total P solubl ein acid (Pts) and organic $(\mathrm{Po})$ and inorganic $(\mathrm{Pi}) \mathrm{P}$ sol ubl ein acid being evaluated. Theother two plants were harvested at the end of the cycle, with their grain production evaluated. The critical levels of P fractions in the bean plants cultivated, in the GH, O, A and GP soils without liming, were, respectively: $P t=2.3,2.2,2.0$ and $1.3 \mathrm{mg} \mathrm{g}^{-1} ; \mathrm{Pts}=110,67,78$ and $63 ; \mathrm{Po}=32$, 19,20 and 21 and $\mathrm{Pi}=77,47,58$ and $43 \mu \mathrm{g} \mathrm{g}^{-1}$. With liming, the critical P fraction levels were: $\mathrm{Pt}=2.8,3.0,3.5$, and $4.2 \mathrm{mg} \mathrm{g}^{-1} ; \mathrm{Pts}=105,96,130$ and 154; $\mathrm{Po}=47,38,42$ and 52 and $\mathrm{Pi}=59,58,88$ and $103 \mu \mathrm{g} \mathrm{g}^{-1}$. Acid phosphatase activity was not a good characteristic of plant nutrition status under conditions of severedeficiency of $P$.
\end{abstract}

Index terms: critical levels, plant nutrition, enzymeactivity.

\section{NTRODUÇÃO}

As plantas absorvem o $\mathrm{P}$ na forma de ortofosfatos que se encontram na solução do solo, sendo a taxa de absorção relacionada com a concentração do P em solução. Como a concentração de $\mathrm{P}$ em solução depende da capacidade de reabastecimento do solo (fator capacidade), o teor de $\mathrm{P}$ na plantas também depende do fator capacidade de $\mathrm{P}$ do solo. $\mathrm{O}$ fator capacidadedeP, ou poder tampão, podeser entendido como a resistência do solo às mudanças da concentração do P em sol ução (fator intensidade - I) quando se adiciona ou se retira $P$ do solo (fator quantidade-Q) (Novais \& Smyth, 1999). Segundo esses autores, ofator capacidade de $\mathrm{P}(\mathrm{Q} / \mathrm{l})$ é definido pelo equilíbrio entre as variações de Q e de I.

A dependência da concentração de $P$ nas plantas do fator capacidade do solo é relatada em vários trabal hos com diferentes culturas e classes de solos (Muniz et al., 1985; Fabres et al., 1987; Fernandes et al., 1998), sendo as menores concentrações verificadas em sol os de mai or fator capacidade de $P$. Por outrolado, em solos de menor fator capacidade, o acúmulo de $\mathrm{P}$ pelas plantas poderá ser importante para a manutenção de seu crescimento, quando, por alguma razão, a disponibilidade de $\mathrm{P}$ do solo for baixa.
As al ter ações na absorção de $P$, em conseqüência dos atributos dos solos, que variam quanto à facilidade de ceder este elemento para as plantas, provocam mudanças nas formas de sua reserva na planta (Bieleski, 1973). Segundo esse autor, em condições de maior disponibilidade de $P$, ocorre seu acúmul o na forma inorgânica nos vacúol os, servindo como reserva. O nível deP inorgânicoémais sensível à disponibilidade do elemento no meio do que os níveis de várias frações orgânicas de P na planta.

Embora os mecanismos envolvidos não sejam ainda total mente explicados, a dinâmica das frações fosfatadas na planta está relacionada com a atividade de fosfatases, enzimas responsáveis pela desfosforilação de moléculas orgânicas. À medida que diminui o suprimento de $\mathrm{P}$ para as plantas, ocorre um aumento da atividade dessas enzimas (Bieleski \& Fergunson, 1983). Vários trabalhos têm relatado a relação entre deficiência de $P$ e atividade de fosfatases (Silva \& Basso, 1993; Ascencio, 1994; Fernandez \& Ascencio, 1994; Fernandes et al., 1998).

Segundo Besford (1979), a determinação da atividade de fosfatases para algumas espécies vegetais pode ser utilizada como uma ferramenta de diagnose nutricional, prevendo deficiência de P nas plantas antes do aparecimento dos sintomas visuais de deficiência. No entanto, para ser utilizada como 
ferramenta de diagnose da nutrição fosfatada, é necessário o estabelecimento de padrões para as diversas espécies de plantas e a parte da planta a ser amostrada (Ascencio, 1994).

Assim, os objetivos destetrabalho foram verificar a influência dos atributos de quatro sol os de várzea sobre a distribuição de algumas frações de fósforo nas fol has de feijoeiro e determinar suas respectivas concentrações críticas, a relação dessas frações com a atividade da fosfatase ácida foliar, na ausência e na presença de calagem, ea relação entrea atividade da fosfataseácida determinada "in vitro" e "in vivo".

\section{MATE RIAL E MÉTODOS}

O experimento foi realizado em condições de casa de vegetação no Departamento de Ciência do Solo da Universidade Federal de Lavras, em vasos plásticos com capaci dade para $4 \mathrm{dm}^{3}$, que continham $3 \mathrm{dm}^{-3}$ de solo. Cultivou-se o feijoeiro (Phaseolus vulgaris L. Cv. Carioca-MG), no período de agosto a novembro de 1997, em amostras de quatro solos de várzea não cultivados anteriormente: Glei Húmico $(\mathrm{GH})$, Orgânico (O), Aluvial (A) eGlei Pouco Húmico
(GP), tendo sido o solo Orgânico artificialmente drenado.

As amostras dos solos, coletadas na camada superficial $(0-20 \mathrm{~cm})$ de uma mesma várzea no município de Lavras (MG), foram destorroadas, secas ao ar e passadas em peneira de $5 \mathrm{~mm}$ de abertura. Foram tomadas subamostras e passadas em peneira de $2 \mathrm{~mm}$ de abertura, constituindo a terra fina seca ao ar para caracterização química, física e mineralógica (Quadro 1).

O experimento foi arranjado em esquema fatorial $4 \times 5 \times 2$, com quatro repetições, sendo os fatores: quatro solos (GH, O, A, GP), cinco doses de P (75, $150,300,500,800 \mathrm{mg} \mathrm{dm}^{-3}$ ) e dois níveis de calagem (com e sem cal cário), em del ineamento inteiramente casualizado, com rodízio semanal da posição dos vasos na casa de vegetação.

As doses de fósforo e cal cário foram definidas em experimentos anteriores (Andrade, 1997). As doses de cal cário para el evar a saturação por bases para $50 \%$ aproximadamente corresponderam a 6,4; 4,6; 4,6 e 13,4 t ha-1, para os solos $G H, O, A$ e GP, respectivamente. Utilizou-se um calcário dolomítico calcinado com $35 \%$ de $\mathrm{CaO}, 14 \%$ de $\mathrm{MgO}$ e Poder Relativo de Neutralização Total de 100\%.

Quadro 1. Principais atributos químicos, físicos e mineralógicos dos solos antes da aplicação dos tratamentos

\begin{tabular}{|c|c|c|c|c|c|c|c|c|c|c|c|c|}
\hline Solo & $\mathrm{pH}^{(1)}$ & $\mathbf{P}^{(1)}$ & $\mathbf{K}$ & $\mathrm{Ca}$ & $\mathbf{M g}$ & Al & $\mathbf{H}+\mathbf{A} \mathbf{I}$ & $\mathbf{T}$ & $\mathbf{m}$ & $\mathbf{v}$ & $\mathbf{P R}^{(2)}$ & $\operatorname{CMAP}^{(2)}$ \\
\hline & & \multicolumn{2}{|c|}{$-m g d m^{-3}-$} & \multicolumn{5}{|c|}{$\mathrm{cmol}_{\mathrm{c}} \mathrm{dm}^{-3}$} & \multicolumn{2}{|c|}{$\longleftarrow \%$} & \multicolumn{2}{|c|}{$\mathrm{mg} \mathrm{L}^{-1}$} \\
\hline $\mathrm{GH}$ & 4,7 & 6,0 & 51 & 0,7 & 0,2 & 1,6 & 13,7 & 15 & 61 & 7 & 1,77 & 2.404 \\
\hline $\mathrm{O}$ & 4,6 & 8,0 & 76 & 1,6 & 1,2 & 0,9 & 9,8 & 13 & 23 & 23 & 16,40 & 1.051 \\
\hline A & 4,7 & 3,0 & 103 & 2,7 & 1,1 & 0,2 & 4,5 & 8,6 & 5 & 47 & 27,33 & 689 \\
\hline GP & 4,5 & 3,0 & 36 & 0,6 & 0,2 & 1,1 & 6,3 & 7,2 & 55 & 12 & 28,00 & 648 \\
\hline
\end{tabular}

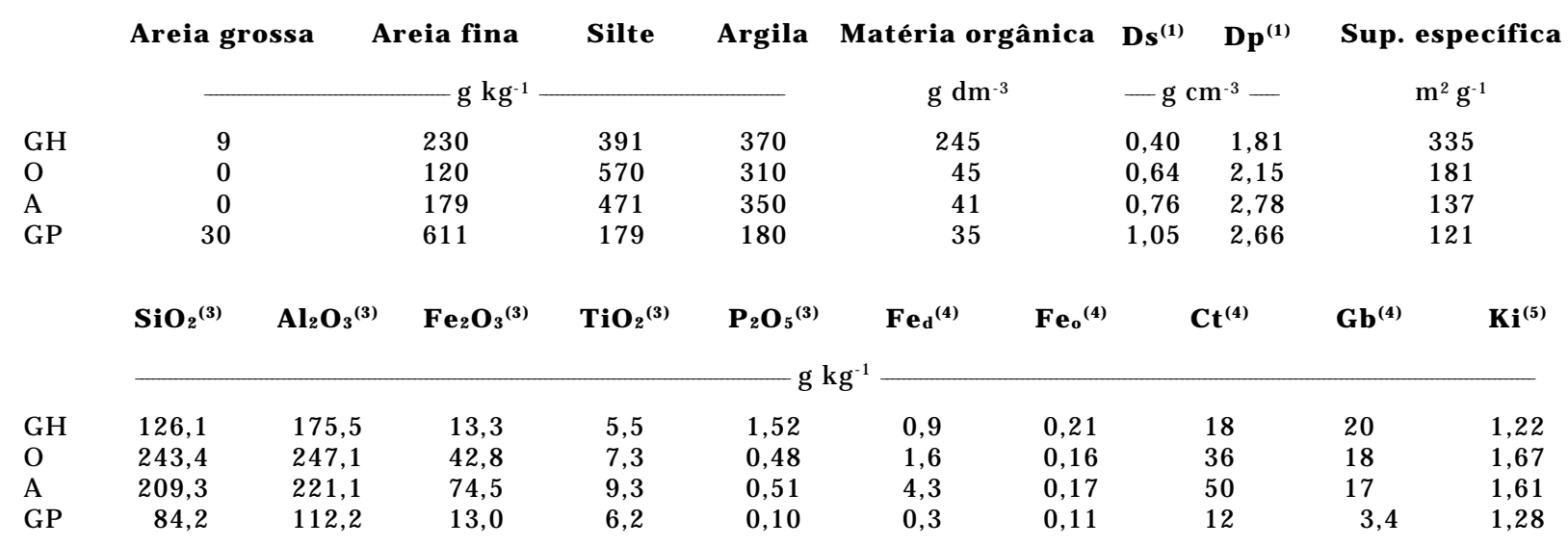

(1) $\mathrm{pH}=\mathrm{pH}$ em água; $\mathrm{P}=$ fósforo pelo Mehlich-1; $\mathrm{T}=$ cap. troca de cátions a $\mathrm{pH}$ 7,0; $\mathrm{m}=$ saturação por $\mathrm{Al}$ e $\mathrm{V}=$ saturação por bases; areia grossa; areia fina; silte; argila; mat. Orgânica; $D s=$ densidade do solo; $D p=$ densidade de partículas (EMBRAPA, 1997). (2) PR $=\mathrm{P}$ remanescente e CMAP = cap. máxima de adsorção de P (Alvarez V. \& Fonseca, 1990). (3) Óxidos do ataque sulfúrico (EMBRAPA, 1997). ${ }^{(4)} \mathrm{Fe}_{\mathrm{d}}=$ ferro ditionito; $\mathrm{Fe}_{\mathrm{o}}=$ ferro oxalato; $\mathrm{Ct}=$ caulinita; $\mathrm{Gb}=$ gibbsita (Mehra \& J ackson, 1960) determinados na fração argila e corrigidos para terra fina. ${ }^{(5)} \mathrm{Ki}$ - relação molecular $\mathrm{SiO}_{2} / \mathrm{Al}_{2} \mathrm{O}_{3}$. 
Os sol os foram incubados nos vasos, com e sem calcário, por um período de 30 dias, mantendo-se a umidade próxima à capacidade de campo. A pós esse período, o material de solo de cada vaso foi seco, peneirado e recebeu a aplicação das doses de $\mathrm{P}$ e de uma adubação básica, com macro e micronutrientes, na forma dereagentes p.a., conformeAndrade(1997): $70 \mathrm{mg}$ de $\left.\mathrm{N}\left(\mathrm{NH}_{4} \mathrm{H}_{2} \mathrm{PO}_{4}, \mathrm{NH}_{4} \mathrm{NO}_{3} \text { e KNO}\right)_{3}\right) ; 100 \mathrm{mg}$ de $\mathrm{K}\left(\mathrm{KNO}_{3}\right) ; 40 \mathrm{mg}$ de $\mathrm{S}\left(\mathrm{CaSO}_{4} \cdot 2 \mathrm{H}_{2} \mathrm{O}\right.$ e $\left.\mathrm{ZnSO}_{4} .7 \mathrm{H}_{2} \mathrm{O}\right) ; 1,5 \mathrm{mg}$ de $\mathrm{Cu}\left(\mathrm{CuCl}_{2} .5 \mathrm{H}_{2} \mathrm{O}\right), 5,0 \mathrm{mg}$ de $\mathrm{Zn}\left(\mathrm{ZnSO}_{4} \cdot 7 \mathrm{H}_{2} \mathrm{O}\right)$ e $1,5 \mathrm{mg}$ de $\mathrm{B}\left(\mathrm{H}_{3} \mathrm{BO}_{3}\right) \mathrm{dm}^{-3}$ de solo. As fontes utilizadas para o fornecimento de $\mathrm{P}$ foram: $\mathrm{NH}_{4} \mathrm{H}_{2} \mathrm{PO}_{4} ; \mathrm{NaH}_{2} \mathrm{PO}_{4}$ e ácido fosfórico. $\mathrm{O}$ material de solo foi novamente incubado por 150 dias, mantendo-se a umidade nos níveis anteriores.

Cultivaram-se três plantas de feijoeiro por vaso, colhendo-se a parte aérea de uma das plantas no florescimento (estádio R6) e das outras duas na maturação fisiológica dos grãos (estádio R9). Durante o cultivo do feijoeiro, a umidade dos sol os foi mantida próxima à capacidade de campo, por mei o de pesagens diárias dos vasos e adição de água deionizada.

Foram realizadas adubações de cobertura com nitrogênio e potássio, diferenciadas de acordo com o crescimento das plantas. Os tratamentos que proporcionaram, em cada solo, um crescimento normal das plantas receberam $150 \mathrm{mg} \mathrm{dm}^{-3}$ de $\mathrm{N}$ e 100 de $K$, parcelados em cinco aplicações. Os tratamentos cujas plantas apresentaram menor crescimento receberam coberturas proporcionalmente menores, $90 \mathrm{mg} \mathrm{dm}^{-3}$ de $\mathrm{N}$ e 60 de K, evitando-se, assim, aplicações excessivas dos nutrientes.

Da planta col hida no florescimento (32 dias após a emergência), coletaram-seamostras dos fol íol os do últimotrifólio fisiologicamente maduro para oestudo do fracionamento do fósforo e da atividade da fosfatase ácida in vivo e in vitro no tecido foliar. No restante das folhas, determinaram-se os teores de fósforo por meio da digestão nítrico-perclórica - $\mathrm{P}$ total (Pt) (Malavolta et al., 1997). Das plantas col hidas no final do ciclo, foi obtido o peso de matéria seca de grãos.

O fracionamento do fósforo foi realizado conforme método de Smille \& Krotkov (1960) e Hogue et al. (1970), modificado por Martinez (1992), obtendo-se as seguintes frações de $\mathrm{P}$ : $\mathrm{P}$ total solúvel em ácido (Pts), $\mathrm{P}$ inorgânico solúvel em ácido $(\mathrm{Pi})$ e, por diferença, $\mathrm{P}$ orgânico ( $\mathrm{Po}$ ). Para determinar a atividade da fosfataseácida in vitro, coletou-se 1,0 g de material vegetal (folíolos), que, depois de recortado em pedaços pequenos $\left( \pm 4 \mathrm{~mm}^{2}\right)$, foi macerado em presença de $0,2 \mathrm{~g}$ de polivinil pirrolidona insolúvel e 0,1 g de ácido ascórbico. Foram adicionados ao material $10 \mathrm{~mL}$ de tampãocitrato 0,1 mol L-1 a pH 4,5, sendo a mistura recolhida em tubos de ensaio e centrifugada a $10.000 \mathrm{rpm}$, por $15 \mathrm{~min}, \mathrm{a} 4^{\circ} \mathrm{C}$. O sobrenadante foi armazenado em freezer. O sedimentofoi ressuspenso em $10 \mathrm{~mL}$ de tampão-citrato $0,1 \mathrm{~mol} \mathrm{L-1}$ a pH 4,5 e centrifugado novamente, e o sobrenadante recol hido no mesmo frasco.

A atividade da fosfatase ácida foi determinada a pH 5,0 (pH ótimo da enzima, determinado em ensaios preliminares), utilizando como substrato o paranitrofenilfosfato a $16 \mathrm{mmol} \mathrm{L}^{-1}$. Em cada determinação, a mistura de reação foi incubada a $30^{\circ} \mathrm{C}$ por quatro diferentes períodos de tempo $(15,30,45$ e $60 \mathrm{~min}$ ). Controles sem enzima (branco de substrato) esem substrato (branco de enzima) foram incubados do mesmo modo que os tubos experimentais (Santos, 1985). Para a determi nação da atividade da fosfatase ácida in vivo, foram retirados discos de $0,5 \mathrm{~cm}$ dos folíol os do último trífólio fisiologicamente maduro, evitando-seas nervuras. O material foi imediatamente transferido para tubos de ensaio depositados em reci piente com gel o e mantidos no escuro. O método utilizado para a determinação da atividadeenzimática foi o descrito por Silva \& Basso (1993) para cana-deaçúcar, onde as amostras de material vegetal são incubadas a $30^{\circ} \mathrm{C}$, por $20 \mathrm{~min}$, com $8 \mathrm{~mL}$ de paranitofenil fosfato $250 \mu \mathrm{mol} \mathrm{L-1}$, em tampão-acetado de sódio 0,1 mol L-1, com pH ajustado para 5,0. Tanto para a atividade da fosfatase ácida determinada in vitro quanto in vivo, as leituras foram realizadas por colorimetria em comprimento de onda de $410 \mathrm{~nm}$.

As variáveis estudadas foram submetidas às análises de variância e de regressão, cujas equações foram ajustadas às médias de produção de matéria seca de grãos conforme as doses de fósforo apl icadas. A partir das equações obtidas, estimaram-seas doses de fósforo para a produção máxima de grãos e $90 \%$ da máxima para cada solo. Para os tratamentos em que foram obtidas equações de regressão linear, adotou-se a maior dose de $\mathrm{P}$ aplicada como aquela necessária para a obtenção da produção máxima e, a partir dessa dose, estimou-se a dose para a obtenção de $90 \%$ da produção máxima.

Foram também ajustadas equações de regressão para o P total e demais frações de P (Pts, Pi e Po) determinadas na planta, na época do florescimento, como variáveis dependentes das doses de fósforo aplicadas. Pela substituição das doses críticas de fósforonessas equações, obtiveram-seas concentrações críticas de diferentes frações de fósforo na parte aérea das plantas de feijoeiro, para cada sol o enível de calagem. Análises de correlações simples foram realizadas entre todas as variáveis estudadas.

\section{RESULTADOS E DISCUSSÃO}

\section{Concentração e níveis críticos foliares de fósforo total}

As concentrações de $\mathrm{P}$ total $(\mathrm{Pt})$ nas fol has das plantas de feijoeiro na época do florescimento 
aumentaram linearmente com as doses de $P$ aplicadas, em todos os tratamentos (Quadro 2). Pelas equações ajustadas, verificou-se que a baixa produção de matéria seca de grãos (MSGR) nos sol os GH eGP na ausência de calagem deveu-seàs baixas concentrações de Pt nas plantas cultivadas nesses solos. No entanto, quando se aplicou calcário, as plantas cultivadas no solo GP foram as que apresentaram as maiores concentrações de Pt, o que pode ser atribuído à menor capaci dade de adsorção do $\mathrm{P}$ aplicado, expressa pelo menor valor de capacidade máxima de adsorção de $\mathrm{P}$ e mai or valor deP remanescentedessesolo (Quadro 1). I nteressante notar, também, que as plantas cultivadas no solo GP foram as que apresentaram a maior resposta em produção de grãos à aplicação de calcário, independentemente das doses de $\mathrm{P}$ aplicadas (Quadro 3). Por outro lado, no GH, que mostrou o maior valor de capacidade máxima de adsorção de $\mathrm{P}$ e menor valor de $\mathrm{P}$ remanescente, as plantas apresentaram as menores concentrações de Pt (Quadro 2).

A capacidade máxima de adsorção e $P$ remanescente são características que refletem o poder tampão ou fator capacidade de $\mathrm{P}$ do solo (Muniz et al., 1985). A variação no fator capacidade entre sol os normal mente está associada à adsorção deP ea al gumas características ligadas ao fenômeno de adsorção, conteúdo e natureza da fração argila e conteúdo de matéria orgânica (Bahia Filho et al., 1983; Muniz et al., 1985). Segundo esses autores, as correl ações significativas entre capaci dade máxima de adsorção, energia de adsorção e $\mathrm{P}$ remanescente são esperadas, tendo em vista a partici pação dessas caracaterísticas na obtenção do fator capacidade. Vários autores observaram relação inversa entre $P$ absorvido pela planta efator capacidade deP do solo (Holford \& Mattingly, 1976; Bahia Filho et al., 1983; Muniz et al., 1985; Fabres et al., 1987; Fernandes et al., 1998).

Verificou-se, ainda, que os acréscimos estimados nos teores de $\mathrm{P}$ para cada unidade de $\mathrm{P}$ aplicada, mostrados pel os coeficientes angulares das equações (Quadro 2), foram maiores quando os solos receberam aplicação de cal cário. Provavel mente, a calagem, além de reduzir a fixação do fósforo aplicado nos solos, reduziu ou eliminou os efeitos do Al sobre a absorção de $\mathrm{P}$ pelas plantas de feijoeiro.

Substituindo nas equações do quadro 2 , para cada solo, na ausência e presença de calagem, as doses correspondentes a $90 \%$ da máxima produção de MSGR (Quadro 3), estimaram-se os níveis críticos de Pt nas fol has das plantas de feijoeiro, na época do florescimento.

$\mathrm{Na}$ ausência de calagem, o solo GP apresentou o menor, e o GH, o maior valor de nível crítico (Quadro 2). O maior valor de nível crítico observado no GH deveu-se à el evada dose de $P$ estimada para $90 \%$ da produção máxima, duas vezes aquela para
Quadro 2. Equações de regressão ajustadas entre fósforo total nas folhas de feijoeiro, em $\mathrm{mg} \mathrm{g}^{-1}$, como variável dependente $(Y)$ das doses de fósforo aplicadas $(X), \mathrm{em} \mathrm{mg} \mathrm{dm}^{-3}$, e níveis críticos foliares de fósforo total para as plantas de feijoeiro

\begin{tabular}{|c|c|c|c|}
\hline Solo & E quação & $\mathbf{R}^{2}$ & $\begin{array}{c}\text { Nível crítico } \\
\text { (mg g-1) }\end{array}$ \\
\hline \multicolumn{4}{|c|}{ Sem calagem } \\
\hline $\begin{array}{l}\mathrm{GH} \\
\mathrm{O} \\
\mathrm{A} \\
\mathrm{GP}\end{array}$ & $\begin{array}{l}Y=0,4551+0,0025^{* * X} X \\
Y=1,2990+0,0027^{* * X} \\
Y=1,2703+0,0022^{* * X} \\
Y=0,7365+0,0014^{* * X}\end{array}$ & $\begin{array}{l}0,95 \\
0,87 \\
0,83 \\
0,94\end{array}$ & $\begin{array}{l}2,3 \\
2,2 \\
2,0 \\
1,3\end{array}$ \\
\hline \multicolumn{4}{|c|}{ Com calagem } \\
\hline $\begin{array}{l}\mathrm{GH} \\
\mathrm{O} \\
\mathrm{A} \\
\mathrm{GP}\end{array}$ & $\begin{array}{l}Y=0,4018+0,0052^{* * X} \\
Y=1,8516+0,0035^{* * X} \\
Y=1,6470+0,0052^{* * X} \\
Y=2,4711+0,0048^{* * X}\end{array}$ & $\begin{array}{l}0,98 \\
0,85 \\
0,94 \\
0,94\end{array}$ & $\begin{array}{l}2,8 \\
3,0 \\
3,5 \\
4,2\end{array}$ \\
\hline
\end{tabular}

** significativo a $1 \%$ pelo teste de $t$.

os demais solos, decorrente do ajuste linear para a produção de MSGR como variável dependente das doses de P aplicadas (Quadro 3). Na presença de calagem, quando o único fator limitante à produção de MSGR foi o P, os solos supostamente de menor fator capacidade, A eGP, apresentaram os maiores valores de níveis críticos (Quadro 2).

Muniz et al . (1985) verificaram grande amplitude de variação entre os níveis críticos de Pt na parte aérea de plantas de soja, tendo sido os menores valores observados para os solos de maior fator capacidade. Segundo Novais \& Smyth (1999), a maior ou menor competição entre planta e solo pelo $P$ aplicado faz com que a planta se ajuste para utilizar o teor de $\mathrm{P}$ que lhe é col ocado à disposição. Dessa forma, quando o $\mathrm{P}$ é o único fator limitante à produção, além das variações decorrentes entre espécies ou cultivares, idade do tecido vegetal, forma do nutriente, interação entre nutrientes e condições ambientais, atributos do solo que refletem o fator capacidade de $\mathrm{P}$ também devem ser considerados na interpretação dos níveis críticos deP em plantas (Muniz et al., 1985).

Correlacionando os níveis críticos de Pt para os quatro solos, com e sem calagem, com alguns atributos dos sol os que refletem seu fator capaci dade de P (Quadro 4), verificaram-se correlações significativas com o teor de argila e de gibbsita, na ausência de calagem, e com o teor de gibbsita, capacidade máxima de adsorção e $\mathrm{P}$ remanescente, na presença de calagem. As correlações com os demais atributos dos solos (Quadro 1) não são apresentadas pelo fato de não se ter verificado tendência de comportamento entre tais atributos e os níveis críticos de $P$. 
Quadro 3. Equações de regressão ajustadas entre a produção de grãos como variável dependente (Y) das doses de fósforo aplicadas (X), produção de grãos correspondentes a $90 \%$ da produção máxima e as doses de P correspondente a $\mathbf{9 0 \%}$ da máxima

\begin{tabular}{|c|c|c|c|c|}
\hline Solo & E quação & $\mathbf{R}^{2}$ & $\begin{array}{c}\text { 90\% produção máxima } \\
\text { de grãos }\end{array}$ & $\begin{array}{l}\text { Dose de } P \text { para } 90 \% \\
\text { da produção máxima }\end{array}$ \\
\hline & & & g vaso-1 & $\mathrm{mg} \mathrm{dm}^{-3}$ \\
\hline \multicolumn{5}{|c|}{ Sem calagem } \\
\hline $\begin{array}{l}\text { GH } \\
\text { O } \\
\text { A } \\
\text { GP }\end{array}$ & $\begin{array}{l}Y=-0,8805+0,0061 * * X \\
Y=-3,01+1,5102 * * X^{0,5}-0,0294 * * X \\
Y=-5,63+1,6838 * * X^{0,5}-0,0343^{* * X} \\
Y=-4,99+0,7547 * * X^{0,5}-0,0145 * * X\end{array}$ & $\begin{array}{l}0,96 \\
0,98 \\
0,99 \\
0,99\end{array}$ & $\begin{array}{r}3,62 \\
14,77 \\
13,55 \\
4,37\end{array}$ & $\begin{array}{l}735 \\
333 \\
322 \\
412\end{array}$ \\
\hline \multicolumn{5}{|c|}{ Com calagem } \\
\hline $\begin{array}{l}\text { GH } \\
\text { O } \\
\text { A } \\
\text { GP }\end{array}$ & $\begin{array}{l}Y=-21,12+2,9811 * * X^{0,5}-0,0541 * * X \\
Y=1,730+1,6894 * X^{0,5}-0,0328 * * X \\
Y=-12,85+3,0192 * * X^{0,5}-0,0604 * * X \\
Y=-9,03+2,7582 * * X^{0,5}-0,0529 * * X\end{array}$ & $\begin{array}{l}0,99 \\
0,98 \\
0,99 \\
0,99\end{array}$ & $\begin{array}{l}17,89 \\
21,14 \\
22,38 \\
24,23\end{array}$ & $\begin{array}{l}460 \\
299 \\
345 \\
359\end{array}$ \\
\hline
\end{tabular}

** significativo a $1 \%$ pelo teste de $t$.

Quadro 4. Coeficientes de correlação linear simples entre as concentrações críticas de fósforo total (Pt), total solúvel em ácido (Pts), orgânico (Po) e inorgânico (Pi) e atributos do solo que refletem o fator capacidade

\begin{tabular}{lcccc}
\hline Atributo do solo & Pt & Pts & Po & Pi \\
\hline & \multicolumn{4}{c}{ Sem calagem } \\
Teor de argila & $0,82^{*}$ & $0,36^{\mathrm{ns}}$ & $0,01^{\mathrm{ns}}$ & $0,46^{\mathrm{ns}}$ \\
Teor de gibbsita & $0,98^{* *}$ & $0,45^{\mathrm{ns}}$ & $0,21^{\mathrm{ns}}$ & $0,54^{\mathrm{ns}}$ \\
CMAP & $0,64^{\mathrm{ns}}$ & $0,92^{*}$ & $0,94^{*}$ & $0,87^{*}$ \\
P-Re & $-0,71^{\mathrm{ns}}$ & $-0,83^{*}$ & $-0,84^{*}$ & $-0,78^{*}$ \\
Matéria orgânica & $0,55^{\mathrm{ns}}$ & $0,95^{*}$ & $0,98^{* *}$ & $0,90^{*}$ \\
& \multicolumn{4}{c}{ Com calagem } \\
Teor de argila & $-0,68^{\mathrm{ns}}$ & $-0,62^{\mathrm{ns}}$ & $-0,80^{*}$ & $-0,53^{\mathrm{ns}}$ \\
Teor de gibbsita & $-0,93^{* *}$ & $-0,93^{* *}$ & $-0,83^{*}$ & $-0,87^{*}$ \\
CMAP & $-0,75^{*}$ & $-0,59^{\mathrm{ns}}$ & $0,07^{\mathrm{ns}}$ & $-0,73^{*}$ \\
P-Re & $0,84^{*}$ & $0,74^{*}$ & $0,09^{\mathrm{ns}}$ & $0,84^{*}$ \\
Matéria orgânica & $-0,65^{\mathrm{ns}}$ & $-0,47^{\mathrm{ns}}$ & $0,20^{\mathrm{ns}}$ & $-0,59^{\mathrm{ns}}$ \\
& &
\end{tabular}

CMAP = capacidade máxima de adsorção de $\mathrm{P} ; \mathrm{P}-\mathrm{Re}=\mathrm{P}$ remanescente. $* *, * \mathrm{e}^{\mathrm{ns}}$ : significativos a $1 \%, 5 \%$ e não-significativo, respectivamente.

Embora o teor de argila seja considerado o atributodo solo demaior importância na determinação do fator capacidade (Miranda \& Vol kweiss, 1981), a ausência de correlação com os níveis críticos de Pt, na presença de calagem (Quadro 4), pode estar relacionada com a natureza da fração argila dos solos estudados. No presente estudo, verificou-se correlação negativa significativa para os teores de gibbsita com os níveis críticos de Pt, possivelmente pela sua alta capacidade em adsorver fósforo.
As concentrações críticas de Pt nas folhas do feijoeiro, estimadas na época do florescimento, correlacionaram-se positiva e significativamente com a produção de MSGR, na ausência e presença de calagem (Quadro 5), indicando constituírem uma boa característica para prever a produção de grãos ea capacidade dos sol os em fornecer $\mathrm{P}$ para as plantas.

\section{Concentrações e níveis críticos foliares de $\mathbf{P}$ total solúvel em ácido, $P$ orgânico e $P$ inorgânico solúvel em ácido}

As concentrações deP total solúvel em ácido (Pts), $\mathrm{P}$ inorgânico $(\mathrm{Pi})$ e $\mathrm{P}$ orgânico $(\mathrm{Po})$ nos tecidos foliares, na época do florescimento do feijoeiro, considerando as doses de $\mathrm{P}$ aplicadas (Quadro 6), apresentaram comportamento semel hante ao verificado para a concentração de Pt nas folhas do feijoeiro.

Comparando as equações ajustadas para Pi e Po e as doses de P aplicadas (Quadro 6), observou-se que os coeficientes angulares, que representam o acréscimo dessas formas de $P$ para cada unidade de $\mathrm{P}$ aplicada, foram sempre maiores para o Pi, indicando que a concentração de Pi é mais sensível às variações de $P$ nos solos. Este comportamento também foi verificado por Fabres et al. (1987), em plantas de alface, e por Furtini Neto et al. (1998), em plantas de eucalipto. Chisholm et al. (1981), trabalhando com Trifolium repens e Stylosanthes hamata, sugeriram que, em vez do Pt, formas de $\mathrm{P}$ metabolicamente ativas, como P-lipídios, deveriam ser utilizadas na determinação de níveis críticos em plantas, uma vez que essas frações são as que menos variam com a idade da planta e com a disponi bilidade do elemento no solo. No entanto, segundo Lee \& Ratcliffe (1983), exatamentepor esteúltimo aspecto, 
Quadro 5. Coeficientes de correlação linear simples entre a produção de matéria seca de grãos (MSGR), fósforo total (Pt), total solúvel em ácido (Pts), orgânico (Po) e inorgânico (Pi) e atividade da fosfatase ácida in vi vo (Pase-vv) e in vitro (Pase-vt), para os solos estudados na ausência e presença de calagem

\begin{tabular}{|c|c|c|c|c|c|c|c|}
\hline & MSGR & Pt & Pts & $\mathbf{P i}$ & Po & Pase-vv & Pase-vt \\
\hline & \multicolumn{7}{|c|}{ Sem calagem } \\
\hline MSGR & $1,00 * *$ & - & - & - & - & - & - \\
\hline $\mathrm{Pt}$ & $0,99 * *$ & $1,00 * *$ & - & - & - & - & - \\
\hline Pts & $0,99 * *$ & $0,99 * *$ & $1,00 * *$ & - & - & - & - \\
\hline $\mathrm{Pi}$ & $0,97 * *$ & $0,98^{* *}$ & $0,99 * *$ & $1,00^{* *}$ & - & - & - \\
\hline Po & $0,99 * *$ & $0,99 * *$ & $0,99 * *$ & $0,99 * *$ & $1,00 * *$ & - & - \\
\hline Pase-vv & $0,95^{* *}$ & $0,92 * *$ & $0,88^{*}$ & $0,85^{*}$ & $0,90^{*}$ & $1,00 * *$ & - \\
\hline \multirow[t]{2}{*}{ Pase-vt } & $0,97 * *$ & $0,94 * *$ & $0,92 * *$ & $0,89 *$ & $0,93^{*}$ & $0,99 * *$ & $1,00 * *$ \\
\hline & \multicolumn{7}{|c|}{ Com calagem } \\
\hline MSGR & $1,00 * *$ & - & - & - & - & - & - \\
\hline $\mathrm{Pt}$ & $0,95 * *$ & $1,00 * *$ & - & - & - & - & - \\
\hline Pts & $0,97 * *$ & $0,99 * *$ & $1,00 * *$ & - & - & - & - \\
\hline $\mathrm{Pi}$ & $0,98 * *$ & $0,99 * *$ & $0,99 * *$ & $1,00 * *$ & - & - & - \\
\hline Po & $0,97 * *$ & $0,99 * *$ & $0,99 * *$ & $0,99 * *$ & $1,00 * *$ & - & - \\
\hline Pase-vv & $-0,42^{\mathrm{ns}}$ & $-0,64^{\mathrm{ns}}$ & $-0,65^{\text {ns }}$ & $0,62^{\text {ns }}$ & $-0,67^{\mathrm{ns}}$ & $1,00 * *$ & - \\
\hline Pase-vt & $-0,54^{\mathrm{ns}}$ & $-0,68^{\text {ns }}$ & $-0,71^{\circ}$ & $-0,69^{\circ}$ & $-0,72^{\circ}$ & $0,98^{* *}$ & $1,00 * *$ \\
\hline
\end{tabular}

**, $*,{ }^{\circ} \mathrm{e}^{\mathrm{ns}}$ significativos a $1,5,10 \%$ e não-significativo, respectivamente.

Quadro 6. Equações de regressão lineares ajustadas entre fósforo total solúvel em ácido (Pts), orgânico (Po) e inorgânico $(\mathrm{Pi})$ solúvel em ácido nas

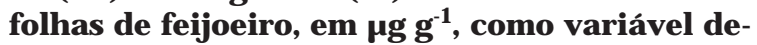
pendente $(Y)$ das doses de fósforo aplicadas $(X)$, em $\mathrm{mg} \mathrm{dm}^{-3}$, eníveis críticos das frações defósforo

\begin{tabular}{|c|c|c|c|c|c|}
\hline Solo & $\begin{array}{c}\text { F ração } \\
\text { de P }\end{array}$ & \multicolumn{2}{|c|}{ E quação } & $\mathbf{R}^{2}$ & $\begin{array}{c}\text { Nível } \\
\text { crítico }\end{array}$ \\
\hline & \multicolumn{4}{|c|}{ Sem calagem } & $\mu \mathrm{g} \mathrm{g}^{-1}$ \\
\hline \multirow[t]{3}{*}{$\mathrm{GH}$} & Pts & $\ddot{\mathrm{Y}}=13,355$ & $+0,0135 * * x$ & 0,93 & 110,0 \\
\hline & $\mathrm{Po}$ & $\hat{\mathrm{Y}}=5,1898$ & $+0,0373 * * x$ & 0,98 & 32,6 \\
\hline & $\mathrm{Pi}$ & $\hat{\mathrm{Y}}=8,3645$ & $+0,0942 * * x$ & 0,90 & 77,4 \\
\hline \multirow[t]{3}{*}{0} & Pts & $\hat{\mathrm{Y}}=21,482$ & $+0,1377 * * x$ & 0,91 & 67,3 \\
\hline & Po & $\hat{\mathrm{Y}}=5,9212$ & $+0,0405^{* *} \mathrm{x}$ & 0,91 & 19,4 \\
\hline & $\mathrm{Pi}$ & $\ddot{\mathrm{Y}}=15,561$ & $+0,0972 * * x$ & 0,89 & 47,9 \\
\hline \multirow[t]{3}{*}{ A } & Pts & $\ddot{\mathrm{Y}}=25,322$ & $+0,1638 * * x$ & 0,93 & 78,1 \\
\hline & Po & $\ddot{\mathrm{Y}}=7,5079$ & $+0,0386 * * x$ & 0,95 & 19,9 \\
\hline & $\mathrm{Pi}$ & $\hat{\mathrm{Y}}=17,815$ & $+0,1252 * * x$ & 0,92 & 58,2 \\
\hline \multirow{3}{*}{ GP } & Pts & $\ddot{\mathrm{Y}}=14,424$ & $+0,1197 * * x$ & 0,94 & 63,7 \\
\hline & Po & $\ddot{\mathrm{Y}}=3,8218$ & $+0,0408 * * x$ & 0,96 & 20,6 \\
\hline & $\mathrm{Pi}$ & $\hat{\mathrm{Y}}=10,603$ & $+0,0789 * * x$ & 0,91 & 43,1 \\
\hline \multicolumn{6}{|c|}{ Com calagem } \\
\hline \multirow[t]{3}{*}{$\mathrm{GH}$} & Pts & $\hat{\mathrm{Y}}=24,289$ & $+0,1768 * * x$ & 0,93 & 105,6 \\
\hline & Po & $\ddot{\mathrm{Y}}=19,059$ & $+0,0609 * * x$ & 0,86 & 47,1 \\
\hline & $\mathrm{Pi}$ & $\mathrm{Y}=5,2308$ & $+0,1159 * * x$ & 0,95 & 58,5 \\
\hline \multirow[t]{3}{*}{$\mathrm{O}$} & Pts & $\ddot{\mathrm{Y}}=31,151$ & $+0,2175^{* *} x$ & 0,91 & 96,2 \\
\hline & Po & $\ddot{\mathrm{Y}}=18,947$ & $+0,0644 * * x$ & 0,91 & 38,2 \\
\hline & $\mathrm{Pi}$ & $\ddot{\mathrm{Y}}=12,20$ & $+0,1531^{* * x}$ & 0,89 & 58,0 \\
\hline \multirow[t]{3}{*}{ A } & Pts & $\ddot{\mathrm{Y}}=51,641$ & $+0,2284 * * x$ & 0,89 & 130,4 \\
\hline & Po & $\ddot{\mathrm{Y}}=25,862$ & $+0,0479 * * x$ & 0,91 & 42,4 \\
\hline & $\mathrm{Pi}$ & $\ddot{\mathrm{Y}}=25,779$ & $+0,1805^{* *} x$ & 0,87 & 88,1 \\
\hline \multirow[t]{3}{*}{ GP } & Pts & $\ddot{\mathrm{Y}}=63,443$ & $+0,2536 * * x$ & 0,91 & 154,5 \\
\hline & Po & $\ddot{\mathrm{Y}}=10,739$ & $+0,0591 * * x$ & 0,86 & 52,0 \\
\hline & $\mathrm{Pi}$ & $\ddot{\mathrm{Y}}=32,704$ & $+0,1945^{* *} \mathrm{x}$ & 0,91 & 102,5 \\
\hline
\end{tabular}

** significativo a $1 \%$ pelo teste t. talvez as frações orgânicas não estimem bem os níveis críticos em plantas, já que essas frações variam muito pouco com o "status" do nutriente na planta.

Nopresenteestudo, verificou-semaior contribuição do Pi em relação ao Po, para o Pts (Figuras 1 e 2). Segundo Bieleski \& Ferguson (1983), em termos quantitativos, em plantas bem supridas em $\mathrm{P}$, o Pi é a fração que mais contribui, geralmente com mais de $50 \%$. No entanto, na presença decalagem, maiores concentrações de $\mathrm{Po}$ em relação ao Pi foram verificadas paras os solos $\mathrm{GH}, \mathrm{O}$ e $\mathrm{A}$ na dose mais baixa de $\mathrm{P}$ (Figura 2), provavelmente por causa da intensa deficiência de fósforo observada para as plantas cultivadas nesses tratamentos. Tal comportamento também foi observado por Hogue et al. (1970) em plantas de tomate deficientes em P.

Pela substituição da dose deP estimada para 90\% da máxima produção de MSGR nas equações da quadro 6, estimaram-se os níveis críticos de $\mathrm{Pts}, \mathrm{Pi}$ ePo. Na ausência decalagem, osoloGH apresentou os maiores níveis críticos de Pts, Pi e Po (Quadro 6). Esse comportamento deveu-se à dose el evada de $\mathrm{P}$ estimada para $90 \%$ da produção máxima, como verificado para o nível crítico de Pt, devido ao ajuste linear entre a MSGR e as doses de P. Na presença decalagem, verificaram-se maiores val ores deníveis críticos de Pts, Pi e Po nos sol os A e GP (Quadro 6), demonstrando menor fator capacidade de $P$. Fernandes et al. (1998) também verificaram maiores valores dessas frações de fósforo em plantas de feijoeiro cultivadas em solos de menor fator 
capacidade, quando corrigi dos com cal cário. Fabres et al. (1987) encontraram comportamento semel hante em plantas de al face cultivadas em amostras de dez Latossolos de diferentes fator capacidade.

Os maiores valores de níveis críticos de $\mathrm{Pi}$ nos solos A e GP estão relacionados com a menor capacidade de adsorção de $\mathrm{P}$ e, provavel mente, com as maiores concentrações de $\mathrm{P}$ lábil desses solos. Segundo Bieleski \& Fergunson (1983), em condições de mai or disponibilidade deP, a planta acumula esse nutriente, na forma inorgânica, nos vacúolos. O Pi vacuolar tem a função de reserva e, se a quantidade de $\mathrm{P}$ absorvida for menor que a sua demanda pela célula, ele retorna ao citoplasma, atendendo à exigência metabólica da célula. Estudos com diversas espécies têm mostrado que a concentração de Pi no citoplasma éregulada pel o seu conteúdo no vacúolo, ocorrendo mudanças acentuadas no conteúdo de Pi das células, quase que exclusivamente no reservatório vacuolar, não havendo alterações significativas no reservatório citoplasmático (Po) (Bieleski, 1973; Lee\& Ratcliffe, 1983).

Neste estudo, na ausência de calagem, os níveis críticos de Pts, Po e Pi apresentaram correlações significativas e positivas com a capacidade máxima de adsorção de P (Quadro 4), comportamento contrário ao esperado, possivelmente pelo efeito negativo doal umínio na absorção deP pelas plantas. $\mathrm{Na}$ presença de calagem, o $\mathrm{Pi}$ correlacionou-se negativa e significativamente com a capacidade máxima de adsorção (Quadro 4), evidenciando, como discutido anteriormente, ser essa fração mais sensível à capacidade de adsorção de $\mathrm{P}$ pelos solos. Ainda na presença de calagem, as frações Pts e Pi correlacionaram-se positiva e significativamente com o P remanescente, indicando poder esta característica ser utilizada no estabelecimento de unidades de solos para a interpretação de níveis críticos em plantas de feijoeiro.

As concentrações críticas das frações de P (Pts, Po ePi) correlacionaram-se positiva esignificativamente com a produção de MSGR, na ausência e presença de calagem (Qaudro 5), evidenciando que essas frações podem ser utilizadas como indicativos da nutrição fosfatada, desde que, como comentado para o Pt, os atributos do solo, relacionados com o fator capacidade deP, sejam considerados.

\section{Atividade da fosfatase ácida}

Para a atividade da fosfataseácida, determinada in vivo ein vitro, a interação sol o x calagem $x$ fósforo foi significativa ( $P<0,01)$. Embora os valores tenham diferido em magnitude, as atividades da fosfatase ácida determinadas in vivo e in vitro apresentaram comportamentos semel hantes nas plantas de feijoeiro cultivadas nos sol os estudados, na ausência e na presença de calagem (Quadro 5), consi derando as doses de P aplicadas (F igura 2). U ma vez que a determinação da atividade da fosfatase ácida in vivo é mais simples e rápida do que a in vitro, a utilização daquela como característica de diagnóstico da nutrição das plantas de feijoeiro em $P$ pode ser usada, a princípio, satisfatoriamente, necessitando de mais estudos.

Esperava-semaior atividade da enzima nas doses mais baixas deP. No entanto, para os solos GH eGP, sem calagem, verificou-se um aumento da atividade da fosfatase ácida com o aumento das doses de $\mathrm{P}$. Para os demais solos, na presença e ausência de calagem, a atividade aumentou da primeira para a segunda dose de $\mathrm{P}$ aplicada, exceto para o solo $\mathrm{O}$, com calagem, em que a atividade aumentou até à terceira dose deP, para depois decrescer. Exceto para o solo GH, em que os teores das frações fosfatadas não foram suficientemente altos para inibir a atividade da fosfatase ácida (Figuras 1 e 2), com o aumento das frações de $P$, houve um decréscimo da atividade. Segundo Reid \& Bieleski (1970), o aumento da atividade da fosfatase ácida parece ocorrer em virtude de um incremento na síntese "de novo" da enzima, cuja formação é inibida pelo íon fosfato por um mecanismo de retroinibição.

A menor atividade verificada nas doses mais baixas de $\mathrm{P}$ aplicadas é devida, provavelmente, às alterações no metabolismo normal das plantas, decorrentes da nutrição fosfatada. $\mathrm{Na}$ época da determinação da atividade da fosfatase ácida, as plantas cultivadas nas doses mais baixas, 75 e $150 \mathrm{mg} \mathrm{dm}^{-3}$ de $\mathrm{P}$, apresentavam sintomas visuais de deficiência de $P$.

Ascencio (1994) verificou maior atividade da fosfatase ácida in vivo em plantas de feijoeiro aos 21 dias de idade, quando foram cultivadas na ausência de $P$, porém, aos 28 dias, a atividade foi menor do que notratamento com $\mathrm{P}$. Comportamento semel hante foi relatado por Fernandez \& Ascencio (1994) em raízes defeijoeiro. Esses autores atribuíram a menor atividade da fosfatase ácida no tratamento sem $P$, em relação ao tratamento com $P$, à menor concentração de proteínas ou ao tempo de resposta ao estresse de $\mathrm{P}$ no interior dos tecidos das plantas. Segundo Ascencio (1994), a idade, o estádio de crescimento, o número de dias sob estresse e o tempo após o início do estresse metabólico de P devem ser considerados para que a atividade da fosfatase seja utilizada como uma ferramenta de diagnose da nutrição fosfatada.

Fernandes et al. (1998) não encontraram diferenças estatísticas entrea atividade da fosfatase ácida em fol has de feijoei ro cultivado com e sem $\mathrm{P}$ e atribuíram esse comportamento a alterações metabólicas decorrentes da deficiência de $\mathrm{P}$ nas plantas cultivadas sem apli cação desse nutriente.

É interessantedestacar quena doseintermediária de $\mathrm{P}$ aplicada, $300 \mathrm{mg} \mathrm{dm}^{-3}$, as plantas ainda responderam à adubação fosfatada quanto à produção de MSGR, embora não apresentassem sintomas visuais de deficiência de P. Nesta dose, a 


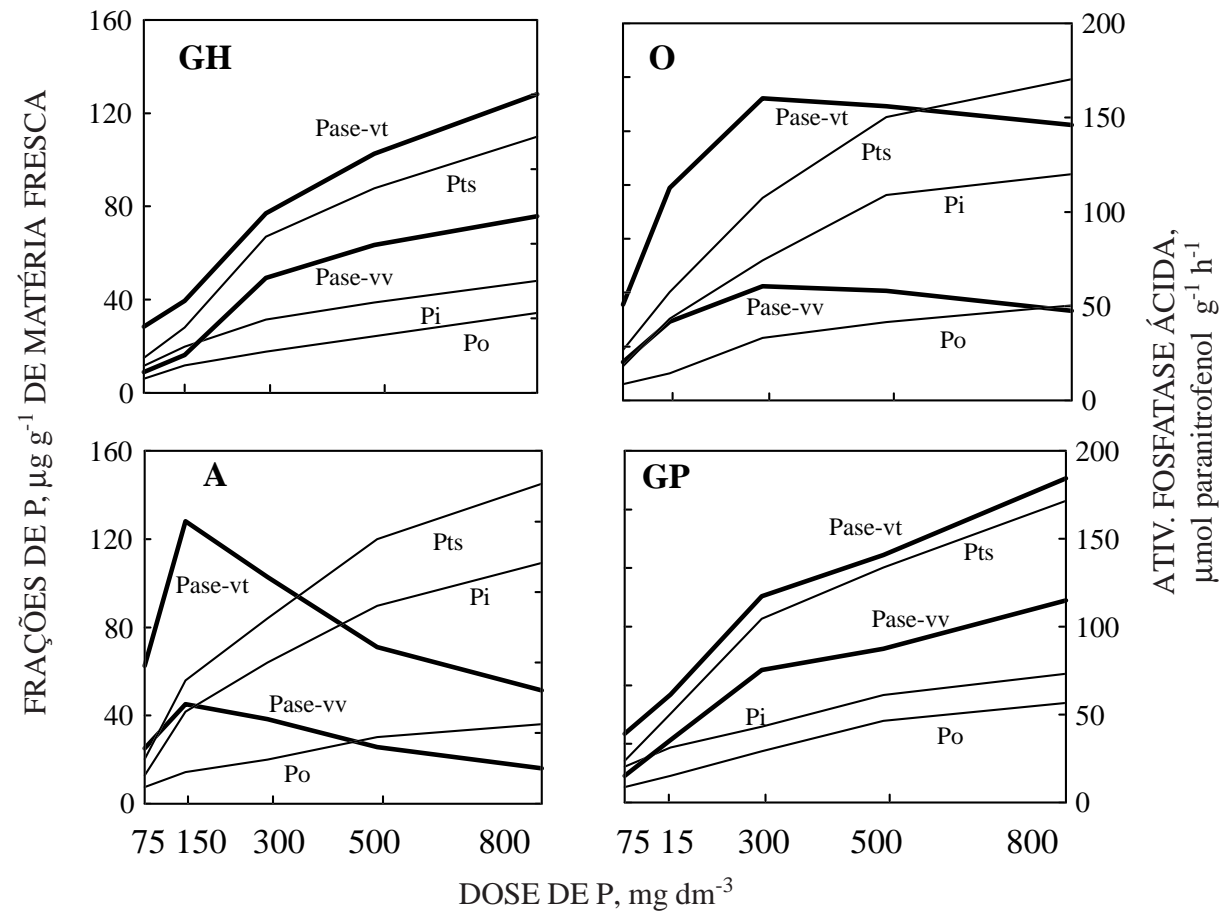

Figura 1. Fósforo total solúvel em ácido (Pts), P orgânico (Po), P inorgânico (Pi) e atividade de fosfatase ácida in vivo (Pase-vv) e in vitro (Pase-vt) nas folhas de feijoeiro cultivado nos solos Glei Húmico (GH), Orgânico, Aluvial (A) e Glei Pouco Húmico (GP), na ausência de calagem.

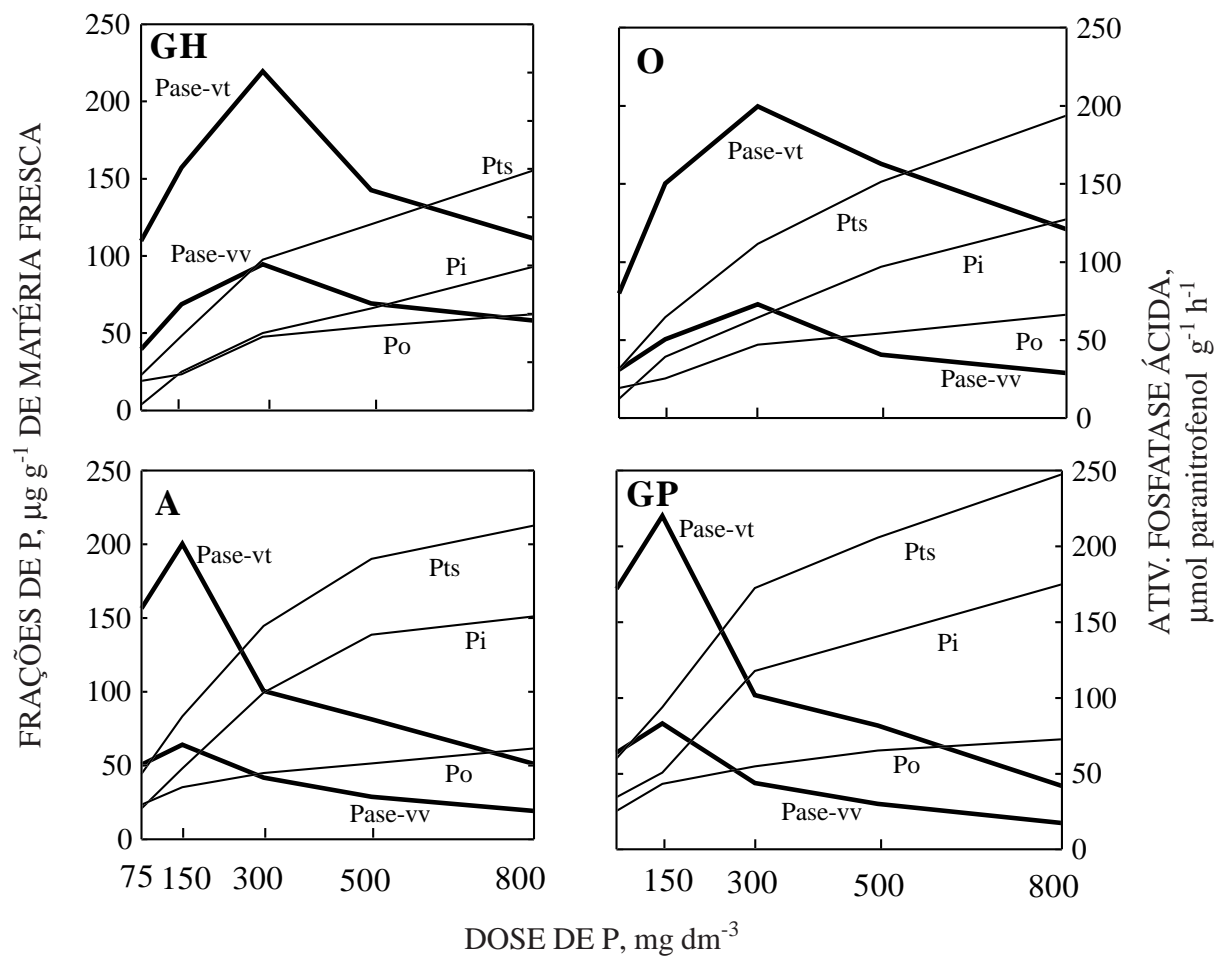

Figura 2. Fósforo total solúvel em ácido (Pts), P orgânico (Po), P inorgânico (Pi) e atividade de fosfatase ácida in vivo (Pase-vv) e in vitro (Pase-vt) nas folhas de feijoeiro cultivado nos solos Glei Húmico (GH), Orgânico, Aluvial (A) e Glei Pouco Húmico (GP), na presença de calagem. 
atividade da fosfatase ácida foi el evada em relação à verificada nas doses de 500 e $800 \mathrm{mg} \mathrm{dm}^{-3}$. Neste caso, em que o P não está numa concentração tão baixa que prejudique os processos metabólicos normais da planta, a determinação da atividade da fosfatase ácida pode ser utilizada como uma ferramenta de diagnose da nutrição fosfatada. Nas doses mais baixas, a fosfatase, para ser utilizada como uma ferramenta de diagnose do estado nutricional das plantas, deve ser determinada, enquando as plantas ainda não apresentarem sintomas visuais de deficiência de $P$.

A atividade da fosfatase ácida in vivo e in vitro, na ausência de calagem, apresentou correlação positiva e significativa com a produção de MSGR e com as frações de P (Quadro 5), indicando que, nessa condição, o aumento da concentração de fósforo na planta aumenta a atividade da fosfatase ácida. $\mathrm{Na}$ presença de calagem, somente a atividade da fosfatase ácida in vitro apresentou correlação significativa, a 10\%, enegativa com o PoePi. A baixa ou a ausência de correlação significativa entre a atividade da fosfatase ácida e a produção de MSGR e as frações de $P$, condição em que o $P$ era o único fator limitante, podem ser explicadas pel o aumento da atividade da enzima com o aumento das doses de $P$, até à segunda ou terceira dose (Figura 2 ), para depois decrescer, nas doses mais el evadas.

\section{CONCLUSÕES}

1. Na presença de calagem, as concentrações das frações de fósforo nos tecidos vegetais das plantas de feijoeiro foram influenciadas pelos atributos do solo, que refletem o poder tampão de fósforo.

2. Os níveis críticos das frações de $P$ estudadas foram diferentes de acordo com o fator capacidade do solo. Dessa forma, em condições de deficiência de $\mathrm{P}$, solos com maior capacidade máxima de adsorção de $\mathrm{P}$, o P orgânico expressou mel hor a concentração crítica do elemento nas folhas, enquanto, em condições de menor deficiência de $\mathrm{P}$, sol os com menor capaci dade máxima de adsorção de P, oP inorgânico foi a fração de mel hor representatividade.

3. A atividade da fosfatase ácida determinada in vivo apresentou comportamento semelhante à in vitro. Na presença de calagem, não se observou correlação significativa para a atividade dessa enzima com a produção de matéria seca de grãos e frações de fósforo nas fol has de feijoeiro.

\section{LITERATURA CITADA}

ALVAREZV., V.H. \& FONSECA, D.B. Definição dedoses defósforo para determinação da capacidade máxima de adsorção de fosfatos e para ensaios em casa de vegetação. R. Bras. Ci. Solo, 14:49-55, 1990.
ANDRADE, C.A. B. Limitações de fertilidade e efeito do cal cário para o feijoei ro em sol os de várzea do sul de Minas Gerais. Lavras, UniversidadeFederal de Lavras, 1997. 107p. (Tese de Doutorado)

ASCENCIO, J . Acid phosphatase as a diagnostic tool. Comm. Soil Sci. Plant Nut., 25:1553-1564, 1994.

BAHIA FILHO, A.F.C.; BRAGA, J .M.; RESENDE, M. \& RIBEIRO, A.C. Relações entre adsorção e componentes mineralógicos da fração argila de Latossol os do Planalto Central. R. Bras. Ci. Solo, 7:221-226, 1983.

BESFORD, R.T. Phosphorus nutrition and acid phosphatase activity in the leaves of seven plant species. J . Sci. Food Agric., 30:281-285, 1979.

BIELESKI, R.L. Phosphate pools, phosphate transport, and phosphate availability. Ann. Res. Plant Phys., 24:225-252, 1973.

BIELESKI, R.L. \& FERGUNSON, J.B. Physiology and metabolism of phosphate and its compounds. In: PIRSON, A. \& ZIMMERMANN, M.H., eds. Encyclopedia of plant physiology: inorganic plant nutrition. Berlin, Spring Verlang, 1983. p.422-4490. (New series, v.15A)

CHISHOLM, R.H.; BLAIR, G.J .; BOWDEN,J .W. \& BOFINGER, V.J . I mproved of "critical" phosphorus concentration from considerations of plant phosphorus chemistry. Comm. Soil Sci. Plant Anal., 12:1059-1065, 1981.

EMPRESA BRASILEIRA DE PESQUISA AGROPECUÁRIA EMBRAPA. Serviço nacional de Levantamento e conservação de solo. Manual de métodos de análise de sol o. Rio de J aneiro, 1997. 212p.

FABRES, A.S.; NOVAIS, R.F.; NEVES, J .C.L.; BARROS, N.F. \& CORDEIRO, A.T. Níveis críticos de diferentes frações de fósforo em plantas de alface cultivadas em diferentes solos. R. Bras. Ci. Solo, 11:51-57, 1987.

FERNANDES, L.A.; FURTINI NETO, A.E.; CURI, N.; GUEDES, G.A.A. \& LI MA, J .M. Fósforo e atividade da fosfatase ácida em plantas de feijoeiro. Pesq. Agropec. Bras., 33:769-778, 1998.

FERNANDEZ, D.S. \& ASCENCIO, J . Acid phosphatase activity in bean and cowpea plants grown under phosphorus stress. J . Plant Nutr., 17:229-241, 1994.

FURTINI NETO, A.E.; BARROS, N.F.; NOVAIS, R.F. \& OLIVEIRA, M.F.G. Frações de fósforo em mudas de Eucalyptus. R. Bras. Ci. Solo, 22:267-274, 1998.

HOGUE, E.; WILCOX, G.E. \& CANTLIFFE, D.J . Effect of soil phosphorus levels on phosphate fractions in tomato leaves. J . Am. Soc. Hort. Sci., 95:174-176, 1970.

HOLFORD, I.C.R. \& MATTINGLY, G.E.G. Effects of phosphate buffering on the labile phosphate by soil test. Aust. J . Soil Res., 17:377-389, 1979.

LEE, R.B. \& RATCLIFFE, R.G. Phosphorus nutrition and the intracellular distribution of inorganic phosphate in pea root tips: a quantitative study using ${ }^{31} \mathrm{P}-\mathrm{RMN}$. J . Exp. Bot., 34:1222-1244, 1983.

MALAVOLTA, E.; VITTI, G.C. \& OLIVEIRA, S.A. Avaliação do estado nutricional das plantas - princípios e aplicações. Piracicaba, Potafos, 1997. 289p. 
MARTINEZ, H.E.P. Cinética da absorção e das frações fosfatadas em setevariedades de soja [Glycinemax L. (Merril)]. Viçosa, Universidade Federal de Viçosa, 1992. não paginado. (Relatório Final de Pós-Doutoramento)

MEHRA, O. P. \& J ACKSON, N. L. I ron oxide removal from soils and clays by a dithionite-citrate system buffered with sodium bicarbonate. Clays Clays Miner., 3:317-327, 1960.

MIRANDA, L.N. \& VOLKWEISS, S.J . Relações entre a resposta da soja a adubação fosfatada e alguns parâmetros do solo. R. Bras. Ci. Solo, 5:58-63, 1981.

MUNIZ, A.S.; NOVAIS, R.F.; BARROS, N.F. \& NEVES, J .C.L. Nível crítico de fósforo na parte aérea da soja como variável do fator capacidade do fósforo. R. Bras. Ci. Solo, 9:237-244, 1985.
NOVAIS, R.F. \& SMYTH, T.J. Fósforo em solo e planta em condições tropicais. Viçosa, U niversidade federal de Viçosa, 1999. 399p.

REID, M.S.M. \& BIELESKI, R.L. Changes in phosphataseactivity in phosphorus deficient Spirodela. Planta, 94:273-284, 1970.

SANTOS, C.D. Fisiologia e bioquímica da digestão em Erinniy ello (Lepidoptera: Sphingidae). São Paulo, Universidade de São Paulo, 1985. 178p. (Tese de Doutorado)

SILVA, F.C. \& BASSO, L.C. Avaliação da atividade in vivo da fosfatase ácida da folha na diagnose da nutrição fosfórica em cana-de-açúcar. R. Bras. Ci. Solo, 17:371-375, 1993.

SMILLE, R.M. \& KROTKOV, G. The estimation of nucleic acids in some algae and higher plants. Can. J. Bot., 38:31-49, 1960. 
L. A. FERNANDES et al.

R. Bras. Ci. Solo, 24:561-571, 2000 